\title{
MATHEMATICAL ASPECTS OF THE PROBLEM OF ACOUSTIC WAVES IN A PLANE STRATIFIED MEDIUM*
}

\author{
G. M. WING \\ Sandia Corporation
}

1. Introduction. In the last few years many papers concerning the problem of acoustic waves in layered media have appeared in the literature. (See, for example, Pekeris, [1, 2], Rudnick [3], and Haskell [4].) While the results obtained have often conformed to the observed physical facts, most of these papers that have come to the author's attention have contained ambiguities that are quite disturbing and confusing from the mathematical viewpoint. This paper has been written to show how a rather old, but often overlooked, device can be used to put these investigations on a firmer mathematical basis.

We consider the specific problem of a point source of acoustic radiation of a single frequency situated above a perfectly reflecting plane surface. The source is in a medium of constant density and sound speed. A plane interface above the source and parallel to the reflector separates this medium from another of different (constant) properties. The second medium extends to infinity. We pose the problem of finding the pressure distribution at any point in the media, concentrating on the pressure on the reflecting surface at large distances from the source.

To avoid the analytical difficulties caused by the infinite geometry, we introduce a small damping term in the wave equation. (For an early mention of this device, see Lamb [8].) The result of this is that functions arising in the analysis are much better behaved than when there is no damping. In the final result, the damping factor is allowed to tend to zero, and the usual pressure behavior is found. In some cases, of course, it may be desirable to examine the result for non-zero damping.

We shall attempt to point out as we go on where difficulties occur in the standard analysis and how they are avoided here.

2. The mathematical model. We introduce a cylindrical coordinate system (see Fig. 1). Medium I is situated between planes $z=0$ and $z=\mathrm{L}$. Medium II is the infinite half-space $z>\mathrm{L}$. The plane $z=0$ is a perfect reflector. Media I and II are characterized by densities $\rho_{1}$ and $\rho_{2}, \rho_{1} \neq \rho_{2}$, and sound speeds $c_{1}$ and $c_{2}, c_{1} \neq c_{2}$, respectively. A pulsating source producing an acoustic wave of frequency $\omega$ is situated at $r=0, z=z_{0}$, $0<z_{0}<\mathrm{L}$, where $r$ is the usual cylindrical radial coordinate. We ask for the pressure at any point in the space $z \geq 0$, concentrating on that at $z=0$, and with $r$ large.

MEDIUM II $z=L$

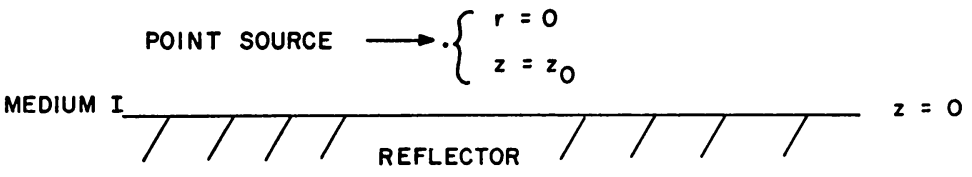

FIg. 1.

*Received July 28, 1960. Revised manuscript received March 10, 1961. 
Since the problem has no $\theta$ dependence, the pressure field may be determined from the potential $\phi=\phi(r, z, t)$. Ordinarily, $\phi$ is given by

$$
\nabla^{2} \phi_{i}=\frac{1}{c_{j}^{2}} \frac{\partial^{2} \phi_{i}}{\partial t^{2}}, \quad j=1,2 ;
$$

where $\phi_{i}$ is the potential function in the $j$ th medium. However, as mentioned in Sec. 1, we choose to introduce a small damping term in (2.1). Thus, we ask that $\phi$ satisfy

$$
\nabla^{2} \phi_{i}=\frac{1}{c_{i}^{2}} \frac{\partial^{2} \phi_{i}}{\partial t^{2}}+\gamma_{i} \frac{\partial \phi_{i}}{\partial t}, \quad j=1,2 ;
$$

where $\gamma_{j},(j=1,2)$ is positive, but small. The condition that $z=0$ be a perfect reflector requires

$$
\frac{\partial \phi_{1}}{\partial z}=0, \quad z=0
$$

while the usual conditions of continuity of pressure and vertical velocity component at $z=\mathrm{L}$ give

$$
\begin{gathered}
\rho_{1} \frac{\partial \phi_{1}}{\partial t}=\rho_{2} \frac{\partial \phi_{2}}{\partial t}, \quad z=L ; \\
\frac{\partial \phi_{1}}{\partial z}=\frac{\partial \phi_{2}}{\partial z}, \quad z=L .
\end{gathered}
$$

In addition, the pressure must be "well-behaved" (at least bounded) as $r^{2}+z^{2} \rightarrow \infty$, a matter to be considered in more detail as we proceed.

Finally, there is a time-dependent source at $r=0, z=z_{0}$. This we take to contain a time factor $e^{i \omega t}$. Since time enters only in this way, we may remove the $t$ dependence in our problem by writing

$$
\phi(r, z, t)=\psi(r, z) e^{i \omega t}
$$

Equations (2.2) become

$$
\begin{aligned}
\nabla^{2} \psi_{i} & +\left(\frac{\omega^{2}}{c_{i}^{2}}-i \omega \gamma_{i}\right) \psi_{i}=0, \quad j=1,2 ; \\
\frac{\partial \psi_{1}}{\partial z} & =0, \quad z=0 \\
\rho_{1} \psi_{1} & =\rho_{2} \psi_{2}, \quad z=L \\
\frac{\partial \psi_{1}}{\partial z} & =\frac{\partial \psi_{2}}{\partial z}, \quad z=L .
\end{aligned}
$$

Equations (2.4), together with the source behavior at $r=0, z=z_{0}$, then describe our problem. We shall often write

$$
k_{i}^{2}=\frac{\omega^{2}}{c_{i}^{2}}-i \omega \gamma_{i}, \quad j=1,2 .
$$

3. The source term. To determine the source contribution to the solution of (2.4) we find the potential $\psi_{s}$ that would occur due to this source in an infinite medium with the properties of medium I. The solution to 


$$
\nabla^{2} \psi_{s}+k_{1}^{2} \psi_{s}=0
$$

which we seek is

$$
\psi_{s}=S \frac{\exp \left(-i k_{1} R\right)}{R},
$$

where $S$ is the source strength and $R$ is the spherical polar coordinate referred to the source, $R=\left(r^{2}+\left|z-z_{0}\right|^{2}\right)^{1 / 2}$. The solution with the $\left(-i k_{1} R\right)$ exponent is chosen instead of the one with $\left(i k_{1} R\right)$ in order that the corresponding $\phi_{s}$ represent an outgoing wave when we choose $k_{1}$ such that $\operatorname{Re}\left(k_{1}\right)>0$. We agree always to take that root of (2.5). Since $\gamma_{i}$ is small we have, approximately,

$$
k_{i} \stackrel{\circ}{=}\left(\omega / c_{i}-\frac{i \gamma_{j} c_{j}}{2}\right),
$$

so that

$$
\phi_{s} \stackrel{\circ}{=} \frac{S}{R} \exp \left[i \omega\left(t-R / c_{1}\right)\right] \exp \left(-\gamma_{1} c_{1} R / 2\right)
$$

Thus $\phi_{s}$ is an attenuated wave, since $\gamma_{1}>0$. Henceforth we assume, for convenience, that $S=1$.

We now transform (3.2) by using the formula (see Watson [6, p. 416])

$$
\frac{\exp \left[-\sigma\left(a^{2}+b^{2}\right)^{1 / 2}\right]}{\left(a^{2}+b^{2}\right)^{1 / 2}}=\int_{0}^{\infty} \frac{t J_{0}(b t)}{\left(t^{2}+\sigma^{2}\right)^{1 / 2}} \cdot \exp \left[-a\left(t^{2}+\sigma^{2}\right)^{1 / 2}\right] d t
$$

holding for $a, b$ non-negative and $|\arg \sigma|<\pi / 2$. Choose $a=\left|z-z_{0}\right|, b=r, \sigma=i k_{1}$. Then

$$
\left|\arg \left(i k_{1}\right)\right| \doteq\left|\arg \left(\frac{\gamma_{1} c_{1}}{2}+\frac{i \omega}{c_{1}}\right)\right|<\frac{\pi}{2},
$$

and we have the representation

$$
\psi_{s}(r, z)=\int_{0}^{\infty} \frac{t J_{0}(r t)}{\left(t^{2}-k_{1}^{2}\right)^{1 / 2}} \exp \left[-\left|z-z_{0}\right|\left(t^{2}-k_{1}^{2}\right)^{1 / 2}\right] d t .
$$

Here the determination of $\left(t^{2}-k_{1}^{2}\right)^{1 / 2}$ chosen is that whose real part is positive.

4. A formal solution to the problem. We now proceed with the formal solution of (2.4). It is customary to apply the Hankel transform [5],

$$
\chi_{i}(\lambda, z)=\int_{0}^{\infty} r J_{0}(\lambda r) \psi_{i}(r, z) d r, \quad j=1,2 .
$$

Consider (2.4) with $j=2$. Multiplying by $J_{0}(\lambda r) r$ and integrating by parts we find, upon recalling Bessel's equation,

$$
\begin{aligned}
\int_{0}^{\infty}\left(\nabla^{2} \psi_{2}+k_{2}^{2} \psi_{2}\right) & J_{0}(\lambda r) r d r \\
= & \left.J_{0}(\lambda r) r \frac{\partial \psi_{2}}{\partial r}\right|_{r=0} ^{r=\infty}+\lambda\left\{\left.r \psi_{2} J_{1}(\lambda r)\right|_{\left.\substack{r=0 \\
r=0}-\lambda \int_{0}^{\infty} r J_{0}(\lambda r) \psi d r\right\}} ^{\infty}\right. \\
& +\int_{0}^{\infty} r \frac{\partial^{2} \psi^{2}}{\partial r^{2}} J_{0}(\lambda r) d r+k_{2}^{2} \chi_{2}=0 .
\end{aligned}
$$


It is well to examine (4.2) carefully before going on. Since [6, p. 199]

$$
J_{,}(\zeta)=\left(\frac{2}{\pi \zeta}\right)^{1 / 2} \cos \left(\zeta-\frac{\pi \nu}{2}-\pi / 4\right)+0\left(\zeta^{-3 / 2}\right)
$$

for large $\zeta$, it is clear that $\psi_{2}$ must be fairly well behaved as $r \rightarrow \infty$ if the formalism of the Hankel transform is to be legitimate. Surely we need at least $r^{1 / 2} \psi_{2} \rightarrow 0$ and $r^{1 / 2} \partial \psi_{2} / \partial r \rightarrow 0$ as $r \rightarrow \infty$.

It is not a priori clear that this is the case. We shall rely temporarily on the fact that the kind of damping we have assumed customarily produces exponentially attenuated solutions, and proceed formally, returning to this whole question at a later point.

Since $\psi_{2}$ and $\partial \psi_{2} / \partial r$ must be continuous at $r=0$ we find, from (4.2),

$$
\frac{d^{2} \chi_{2}}{d z^{2}}+\left(k_{2}^{2}-\lambda^{2}\right) \chi_{2}=0
$$

making the assumption that the interchange of integration and differentiation with respect to $z$ is valid.

The case of $\psi_{1}$ is a little different from that of $\psi_{2}$ since $\psi_{1}$ is admittedly singular at $r=0, z=z_{0}$ because of the point source. However, if we define $\psi_{1}^{*}$ by

$$
\psi_{1}=\psi_{1}^{*}+\psi_{s},
$$

then $\psi_{1}^{*}$ is as well behaved as $\psi_{2}$.

We may now use the standard formal techniques [see 5] to argue that

$$
\begin{aligned}
\psi_{2}(r, z)= & \int_{0}^{\infty} F_{2}(\lambda) \exp \left[-z\left(\lambda^{2}-k_{2}^{2}\right)^{1 / 2}\right] J_{0}(\lambda r) \lambda d \lambda \\
& \quad+\int_{0}^{\infty} G_{2}(\lambda) \exp \left[z\left(\lambda^{2}-k_{2}^{2}\right)^{1 / 2}\right] J_{0}(\lambda r) \lambda d \lambda, \\
\psi_{1}(r, z)=\psi_{0}+\int_{0}^{\infty} F_{1}(\lambda) \exp \left[-z\left(\lambda^{2}-k_{1}^{2}\right)^{1 / 2}\right] J_{0}(\lambda r) \lambda d \lambda & \\
& +\int_{0}^{\infty} G_{1}(\lambda) \exp \left[z\left(\lambda^{2}-k_{1}^{2}\right)^{1 / 2}\right] J_{0}(\lambda r) \lambda d \lambda,
\end{aligned}
$$

where $G_{1}, G_{2}, F_{1}, F_{2}$, are as yet undetermined. However, it must be noted that we have used, in obtaining (4.6), the classical inversion formula for the Hankel transform. A sufficient condition for applicability of this formula is the convergence of

$$
\int_{0}^{\infty} r^{1 / 2}\left|\psi_{j}(r, z)\right| d r, \quad j=1,2 .
$$

This, again, may be expected because of the damping term.

In (4.6), and in all the analysis that follows, we must agree on a determination for $\left(\lambda^{2}-k_{i}^{2}\right)^{1 / 2}, j=1,2$. We shall take that branch whose real part is positive for $\lambda$ real. For $\lambda$ complex, $\operatorname{Re}(\lambda) \geq 0$, we cut the plane as shown in Fig. 2. This is compatible with (3.6).

Now we may apply $(2.4 \mathrm{~b})-(2.4 \mathrm{~d})$, together with the condition that $\psi_{2}$ be at least bounded as $z \rightarrow \infty$ to determine $F_{i}$ and $G_{i}, j=1,2$. The details are lengthy and we shall omit them. Suffice it to say that the final expressions for $\psi_{1}$ and $\psi_{2}$ are 


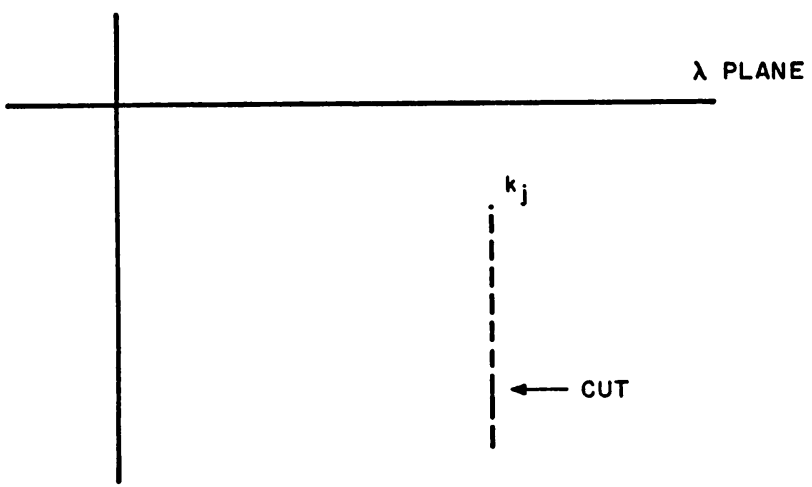

FIG. 2.

$$
\begin{aligned}
\psi_{1}(r, z) & =\int_{0}^{\infty} \frac{\lambda J_{0}(\lambda r)}{\left(\lambda^{2}-k_{1}^{2}\right)^{1 / 2}} \exp \left[-\left|z-z_{0}\right|\left(\lambda^{2}-k_{1}^{2}\right)^{1 / 2}\right] d \lambda \\
+ & \int_{0}^{\infty} \frac{\lambda J_{0}(\lambda r)}{\left(\lambda^{2}-k_{1}^{2}\right)^{1 / 2}} \exp \left[-\left|z+z_{0}\right|\left(\lambda^{2}-k_{1}^{2}\right)^{1 / 2}\right] d \lambda \\
+ & 2 \int_{0}^{\infty}\left[D\left(\lambda ; k_{1}, k_{2}\right)\right]^{-1} \lambda J_{0}(\lambda r) \exp \left[-L\left(\lambda^{2}-k_{1}^{2}\right)\right]\left(1-\frac{\rho_{1}\left(\lambda^{2}-k_{2}^{2}\right)^{1 / 2}}{\rho_{2}\left(\lambda^{2}-k_{1}^{2}\right)^{1 / 2}}\right) \\
& \cdot \cosh \left[z_{0}\left(\lambda^{2}-k_{1}^{2}\right)^{1 / 2}\right] \cosh \left[z\left(\lambda^{2}-k_{1}^{2}\right)^{1 / 2}\right] d \lambda, \\
\psi_{2}(r, z)= & \frac{2 \rho_{1}}{\rho_{2}} \int_{0}^{\infty}\left[D\left(\lambda ; k_{1}, k_{2}\right)\right]^{-1} \lambda J_{0}(\lambda r) \exp \left[-L\left(\lambda^{2}-k_{1}^{2}\right)^{1 / 2}\right]\left(\lambda^{2}-k_{2}^{2}\right)^{1 / 2} \\
\cdot & \left.\cdot \sinh \left[L\left(\lambda^{2}-k_{1}^{2}\right)^{1 / 2}\right]+\cosh \left[L\left(\lambda^{2}-k_{1}^{2}\right)^{1 / 2}\right]\right\} \\
\cdot & \exp \left[-(L-z)\left(\lambda^{2}-k_{2}^{2}\right)^{1 / 2}\right] \cosh \left[z_{0}\left(\lambda^{2}-k_{1}^{2}\right)^{1 / 2}\right] d \lambda,
\end{aligned}
$$

where

$$
\begin{aligned}
D\left(\lambda ; k_{1}, k_{2}\right)=\left(\lambda^{2}-k_{1}^{2}\right)^{1 / 2} \sinh \left[L \left(\lambda^{2}-\right.\right. & \left.\left.k_{1}^{2}\right)^{1 / 2}\right] \\
& \quad+\frac{\rho_{1}}{\rho_{2}}\left(\lambda^{2}-k_{2}^{2}\right)^{1 / 2} \cosh \left[L\left(\lambda^{2}-k_{2}^{2}\right)^{1 / 2}\right] .
\end{aligned}
$$

We shall be especially interested in

$$
\begin{aligned}
\psi_{1}(r, 0)=2 & \int_{0}^{\infty} \frac{\lambda J_{0}(\lambda r) \exp \left[-z_{0}\left(\lambda^{2}-k_{1}^{2}\right)^{1 / 2}\right]}{\left(\lambda^{2}-k_{1}^{2}\right)^{1 / 2}} d \lambda+2 \int_{0}^{\infty}\left[D\left(\lambda ; k_{1}, k_{2}\right)\right]^{-1} \lambda J_{0}(\lambda r) \\
& \cdot \exp \left[-L\left(\lambda^{2}-k_{1}^{2}\right)^{1 / 2}\right]\left(1-\frac{\rho_{1}\left(\lambda^{2}-k_{2}^{2}\right)^{1 / 2}}{\rho_{2}\left(\lambda^{2}-k_{1}^{2}\right)^{1 / 2}}\right) \cosh \left[z_{0}\left(\lambda^{2}-k_{1}^{2}\right)^{1 / 2}\right] d \lambda .
\end{aligned}
$$

5. Discussion of the formal solution. As has been mentioned in several places, the results thus far obtained must be considered as purely formal. The validity of (4.7) could be established by studying the convergence properties of the integrals, carrying out various differentiations, and then checking to determine whether Eqs. (2.4) are indeed satisfied. This would guarantee that we have found a solution to our problem. The details necessary to justify these manipulations are essentially covered in suggestions regarding the treatment of (4.9), on which we now concentrate. 
The first integral in (4.9) represents the contribution of the source and its mirror image, the latter arising because of the perfect reflection at $z=0$. We may focus further attention on the second integral, which we call $2 K$. It is clear that an understanding of the behavior of the denominator $D$ is essential.

We choose to consider $D$ as a function of a complex variable $\lambda$. As such, it is analytic everywhere save at $\lambda= \pm k_{2}$, where there are branch points. To study the zeros of $D$ we initially consider the case $k_{1}$ and $k_{2}$ real, arising from $\gamma_{1}=\gamma_{2}=0$, which implies no damping. We write $k_{1}(0)$ and $k_{2}(0)$ to indicate this, and examine two cases.

Case I

$$
0<k_{1}(0)<k_{2}(0)<\infty ; \text { or } c_{1}>c_{2} .
$$

The first term in $D$ is always real for real $\lambda$. The second is pure imaginary or zero for $0 \leq \lambda<k_{2}(0)$. If this term is zero then $\cosh \left\{L\left[\lambda^{2}-k_{1}^{2}(0)\right]^{1 / 2}\right\}=0$. But then sinh $\left\{L\left[\lambda^{2}-k_{1}^{2}(0)\right]^{1 / 2}\right\} \neq 0$ and $D \neq 0$. For $\lambda=k_{2}(0)$ the first term is positive, while for $\lambda>k_{2}(0)$ both terms in $D$ are positive.

Hence $D$ has no real zeros in Case I.

Case II

$$
0<k_{2}(0)<k_{1}(0)<\infty ; \text { or } c_{1}>c_{2} .
$$

Here the arguments are similar for $0 \leq \lambda<k_{2}(0)$, and for $k_{1}(0) \leq \lambda<\infty$. However, the situation for $k_{2}(0) \leq \lambda<k_{1}(0)$ is quite different. In fact, we find that $D$ has zeros where

$$
\begin{aligned}
\tan (L \beta) & =\frac{\rho_{1}\left[\left(k_{1}^{2}(0)-k_{2}^{2}(0)\right)-\beta^{2}\right]^{1 / 2}}{\rho_{2} \beta}, \\
\beta^{2} & =k_{1}^{2}(0)-\lambda^{2} .
\end{aligned}
$$

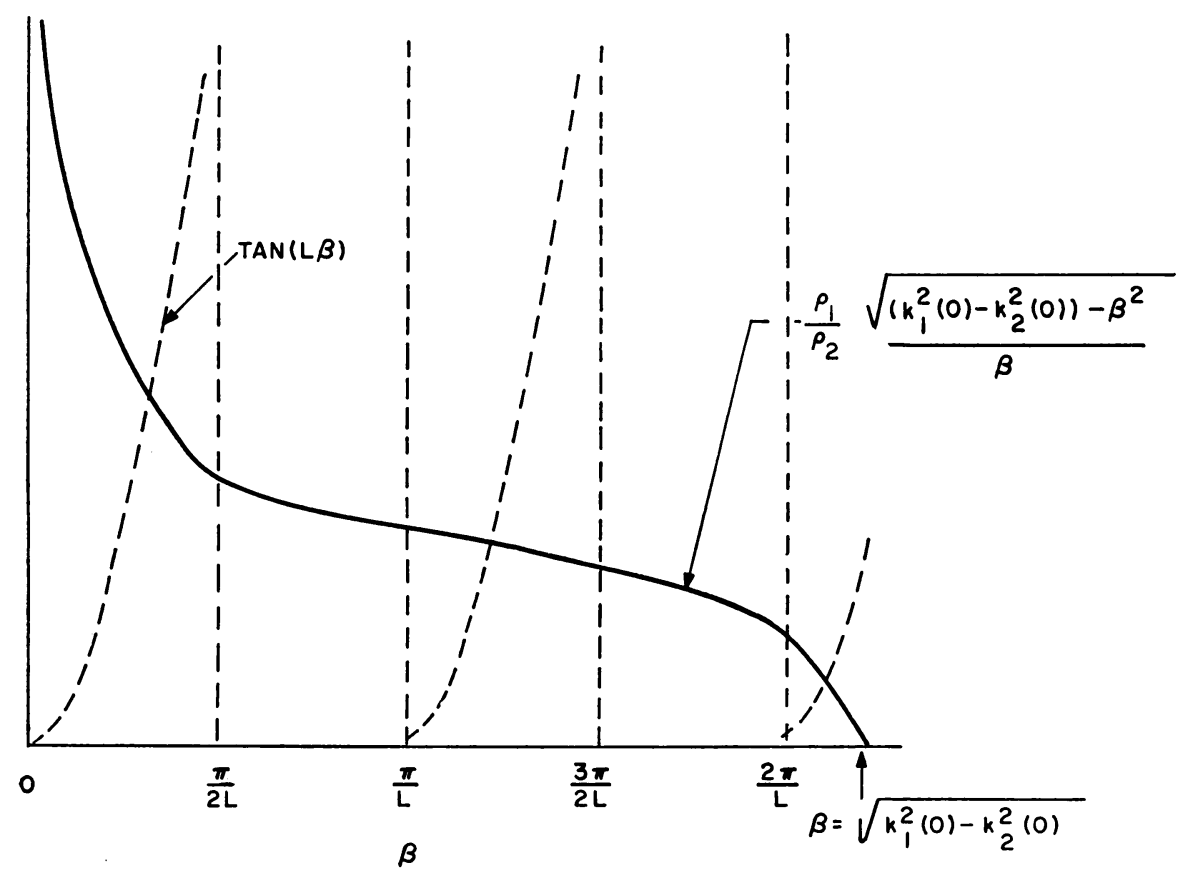

FIG. 3. 
That (5.1) has at least one but not more than a finite number of real solutions is readily seen from Fig. 3.

Hence when $\gamma_{1}=\gamma_{2}=0$ and $k_{2}(0)<k_{1}(0)$, Eqs. (4.7) and (4.9) are quite meaningless as they stand since $D$ has zeros on the path of integration. This is a difficulty which arises in the usual treatment of such acoustic problems as ours. Ordinarily an interpretation is given to the integrals, which, it eventually develops, is physically satisfactory. The mathematical ambiguity is directly connected with the fact that the Hankel transform in its classical form cannot be applied to our problem in the undamped case.

If, however, we take $\gamma_{1}$ and $\gamma_{2}$ small but positive the trouble vanishes automatically. To see this we study the function

$$
\begin{aligned}
D^{*}\left(\lambda ; k_{1}, k_{2}\right)=\left\{\tan \left[L\left(k_{1}^{2}-\lambda^{2}\right)^{1 / 2}\right]\right. & -\frac{\left.\rho_{1}\left(\lambda^{2}-k_{2}^{2}\right)^{1 / 2}\right\}}{\left.\rho_{2}\left(k_{1}^{2}-\lambda^{2}\right)^{1 / 2}\right\}} \\
& \cdot\left\{\tan \left[L\left(k_{1}^{2}-\lambda^{2}\right)^{1 / 2}\right]+\frac{\rho_{1}\left(\lambda^{2}-k_{2}^{2}\right)^{1 / 2}}{\rho_{2}\left(k_{1}^{2}-\lambda^{2}\right)^{1 / 2}}\right\} \\
= & \tan ^{2}\left[L\left(k_{1}^{2}-\lambda^{2}\right)^{1 / 2}\right]-\left(\frac{\rho_{1}}{\rho_{2}}\right)^{2}\left(\frac{\lambda^{2}-k_{2}^{2}}{k_{1}^{2}-\lambda^{2}}\right),
\end{aligned}
$$

which has the advantage over $D$ of being single valued. Consider Case II and let $\lambda_{0}$ be a real zero of $D$. Then

$$
D^{*}\left(\lambda_{0} ; k_{1}(0), k_{2}(0)\right)=0 .
$$

Let $k_{1}$ and $k_{2}$ now have small negative imaginary parts $\epsilon_{1}$ and $\epsilon_{2}$ resulting from a small positive damping factor. Then, since $D^{*}$ is analytic in a region containing $\lambda_{0}$ but avoiding $k_{1}(0)$ and poles of the tangent, we may apply the theorem of Hurwitz on the zeros of a convergent sequence of analytic functions (Titchmarsh [7, p. 119]) and assert that $\lambda_{0}$ will change slightly to $\lambda_{0}+(\sigma+i \tau)$. Thus, to first order in $\epsilon_{1}$ and $\epsilon_{2}$,

$$
\begin{aligned}
D^{*}\left(\lambda_{0}+(\sigma+i \tau) ; \quad k_{1}(0)+\epsilon_{1} i,\right. & \left.k_{2}(0)+\epsilon_{2} i\right) \\
= & (\sigma+i \tau) \frac{\partial D^{*}}{\partial \lambda}+\epsilon_{1} i \frac{\partial D^{*}}{\partial k_{1}}+\epsilon_{2} i \frac{\partial D^{*}}{\partial k_{2}}=0,
\end{aligned}
$$

where the partial derivatives are all evaluated at $\lambda_{0}, k_{1}(0), k_{2}(0)$. It is readily seen that

$$
\frac{\partial D^{*}}{\partial \lambda}<0, \quad \frac{\partial D^{*}}{\partial k_{1}} \geq 0, \quad \frac{\partial D^{*}}{\partial k_{2}}>0 .
$$

Hence $\tau<0$, proving that the real zeros of $D$ move below the axis when small damping is included.

It follows also from Hurwitz's theorem that other roots of $D$ cannot at the same time move onto the real axis. Further, the roots indicated in Fig. 3 are clearly simple. They must, by Hurwitz's theorem, remain that way when $\gamma_{1}>0$ and $\gamma_{2}>0$ are introduced.

It is now clear that the integrals (4.7) are meaningful when damping is considered. Their convergence properties are easily ascertained. We turn now to the asymptotic evaluation of (4.9) for large $r$.

6. An asymptotic estimate of $\psi_{1}(r, 0)$. Our attention focuses on the second integral, $2 K$, of $(4.9)$. It is convenient to write

$$
J_{0}(\lambda r)=\frac{1}{2}\left\{H_{0}^{(1)}(\lambda r)+H_{0}^{(2)}(\lambda r)\right\},
$$


where $H_{0}^{(j)}(\lambda r), j=1,2$, are the Hankel functions as defined in [6, p. 73]. Of particular importance for us are the results [6, p. 170 and p. 211]

$$
\begin{array}{ll}
H_{0}^{(1)}(\xi)=\frac{2}{i \Gamma^{2}\left(\frac{1}{2}\right)} \int_{1}^{\infty} \frac{e^{i \xi t}}{\left(t^{2}-1\right)^{1 / 2}} d t, & \operatorname{Im}(\xi) \geq 0, \\
H_{0}^{(2)}(\xi)=\frac{-2}{i \Gamma^{2}\left(\frac{1}{2}\right)} \int_{1}^{\infty} \frac{e^{-i \xi t}}{\left(t^{2}-1\right)^{1 / 2}} d t, & \operatorname{Im}(\xi) \leq 0 ;
\end{array}
$$

and

$$
\begin{array}{ll}
\left|H_{0}^{(1)}(\xi)\right| \leq G\left|\xi^{-1 / 2} e^{i \xi}\right|, & \operatorname{Re}(\xi) \geq 0, \\
\left|H_{0}^{(2)}(\xi)\right| \leq G\left|\xi^{-1 / 2} e^{-i \xi}\right|, & \operatorname{Re}(\xi) \geq 0,
\end{array}
$$

where $G$ is a positive constant.

First consider for $R$ fixed, $R>\max \left\{\left|k_{1}\right|,\left|k_{2}\right|\right\}$, but otherwise arbitrary,

$$
K(R)=\int_{R}^{\infty} \lambda J_{0}(\lambda r) \frac{N\left(\lambda ; k_{1}, k_{2}\right)}{D\left(\lambda ; k_{1}, k_{2}\right)} d \lambda,
$$

where

$$
N\left(\lambda ; k_{1}, k_{2}\right)=\exp \left[-L\left(\lambda^{2}-k_{1}^{2}\right)^{1 / 2}\right]\left(1-\frac{\rho_{1}\left(\lambda^{2}-k_{2}^{2}\right)^{1 / 2}}{\rho_{2}\left(\lambda^{2}-k_{1}^{2}\right)^{1 / 2}}\right) \cosh \left[z_{0}\left(\lambda^{2}-k_{1}^{2}\right)^{1 / 2}\right] .
$$

Using the estimate (4.3) with $\nu=0$ it is easily seen that

$$
|K(R)|<F(R) r^{-3 / 2} \text {, }
$$

where $F$ is independent of $k_{1}$ and $k_{2}$ for small $\gamma_{1}, \gamma_{2}$.

We are now left, using (6.1), with the study of

$$
K_{i}(R)=\frac{1}{2} \int_{0}^{R} \lambda H_{0}^{(i)}(\lambda r) \frac{N\left(\lambda ; k_{1}, k_{2}\right)}{D\left(\lambda ; k_{1}, k_{2}\right)} d \lambda,
$$

with $j=1,2$.

We concentrate on $K_{2}$. The integrand is not singular on the real axis. The branch lines, according to our agreement in Sec. 4, lie below the real axis. We consider the contour shown in Fig. 4.

Obviously,

$$
-\int_{\delta}^{R}+\sum_{i=1}^{11} \oint_{c_{i}}=2 \pi i \sum \text { Residues. }
$$

First, it may be shown that

$$
\left|D\left(\lambda ; k_{1}, k_{2}\right)\right|>M e^{L \mathrm{Ro}(\lambda)}
$$

provided we choose $R=2 n \pi+\pi / 4, n$ large. The details are messy but elementary and we omit them. In (6.9) $M$ is independent of $k_{1}, k_{2}$, and $n$.

On $C_{11}, \operatorname{Re}\left[\left(\lambda^{2}-k_{1}^{2}\right)^{1 / 2}\right]>0$, and therefore the integrand, exclusive of $H_{0}^{(2)}(\lambda r)$, is bounded on this contour. Hence, using (6.3), we have

$$
\left|\int_{C_{11}}\right| \leq m \int_{0}^{\pi / 2} e^{-r R \sin \theta}(r R)^{-1 / 2} R d \theta \leq m^{\prime} R^{-1 / 2} r^{-3 / 2},
$$

where $m, m^{\prime}$, etc. henceforth represent constants independent of $k_{1}, k_{2}$, and $R$. 


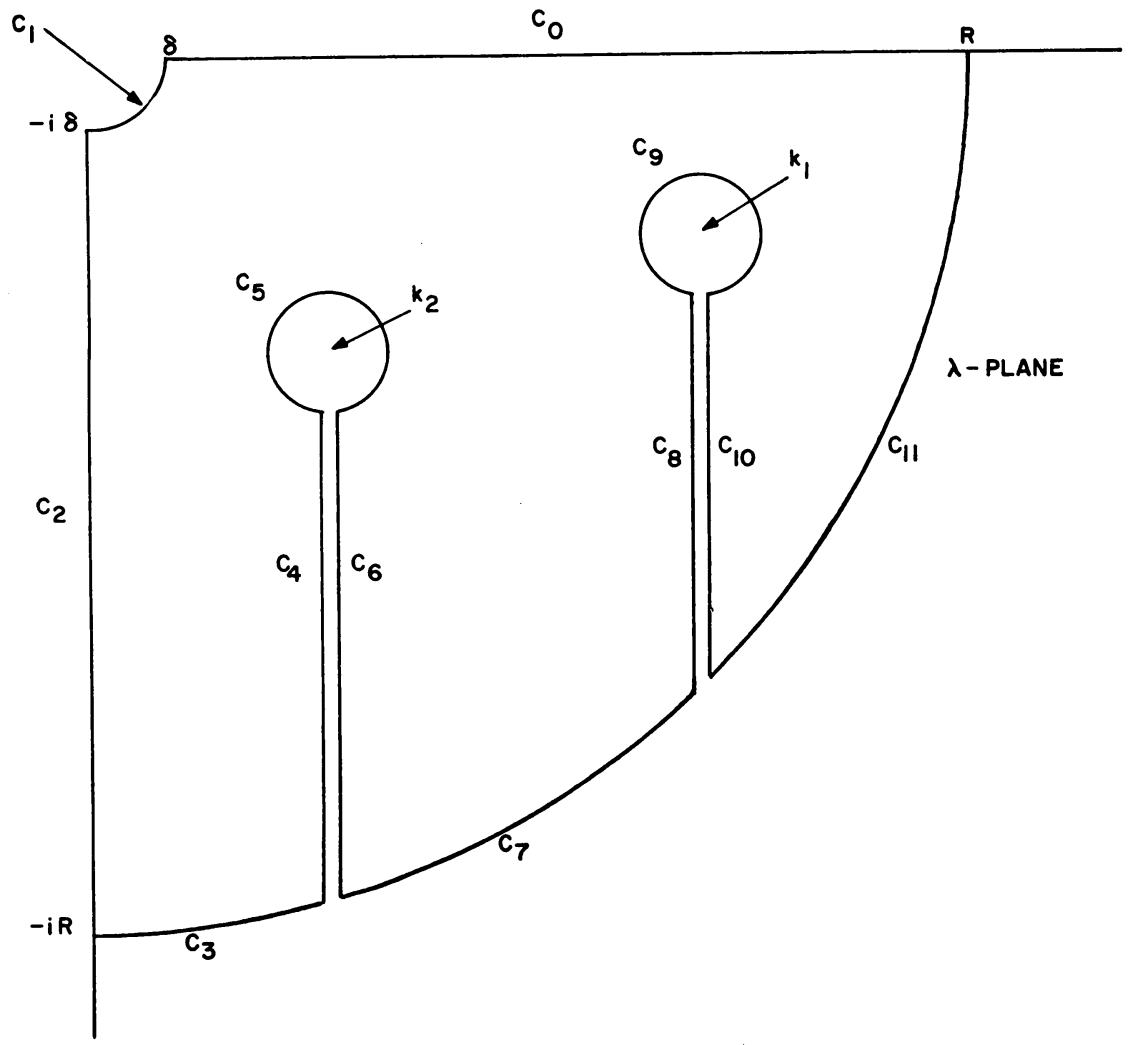

Fig. 4.

On $C_{3}$ and $C_{7}$ it is no longer true that $\operatorname{Re}\left[\left(\lambda^{2}-k_{1}^{2}\right)^{1 / 2}\right]>0$, since the contour has gone around the branch point $k_{1}$. However, for these contours we have, for large $R$,

$$
-\operatorname{Re}\left[\left(\lambda^{2}-k_{1}^{2}\right)^{1 / 2}\right]<2 \operatorname{Re}\left(k_{1}\right),
$$

so that the same kind of estimate holds as in (6.10).

On $C_{2}$ we use (6.2).

$$
\left|\int_{c_{3}}\right| \leq m^{\prime \prime} \int_{0}^{R} d y \int_{1}^{\infty} \frac{e^{-y r t}}{\left(t^{2}-1\right)^{1 / 2}} d t \leq \frac{m^{\prime \prime \prime}}{r} .
$$

For $C_{8}$ we assume that no zero of $D$ occurs on or near $C_{8}$. If it does we distort the contour slightly. Then

$$
\int_{c_{\mathbf{v}}}=\int_{c_{\mathbf{v}}} \frac{P\left(\lambda ; k_{1}, k_{2}\right)}{\left(\lambda^{2}-k_{1}^{2}\right)^{1 / 2}} H_{0}^{(2)}(\lambda r) d \lambda,
$$

where $|P|<\eta(R)$, independent of $k_{1}$ and $k_{2}$. From this we easily see, using $\lambda=e^{3 \pi i / 2} y+k_{1}$, that

$$
\left|\int_{c_{.}}\right| \leq \frac{\eta^{\prime}(R)}{r}
$$

A similar estimate holds for $C_{10}$. 
The calculations along other contours are quite similar, and we may leave them to the reader. To summarize,

$$
K_{2}(R)=-2 \pi i \sum \text { Residues }+\frac{A_{2}\left(R, r ; \gamma_{1}, \gamma_{2}\right)}{r},
$$

where $A_{2}$ is bounded as a function of $r, \gamma_{1}$, and $\gamma_{2}$.

The integration of $K_{1}$ is accomplished by using a path in the upper half plane. The manipulations are easier than for $K_{2}$ and we have

$$
K_{1}(R)=2 \pi i \sum \text { Residues }+\frac{A_{1}\left(R, r ; \gamma_{1}, \gamma_{2}\right)}{r} .
$$

Our work in Sec. 5 has shown that there are certain simple poles with small imaginary parts in the lower right half plane. We designate these by $\lambda_{j}=\nu_{j}-i \mu_{i}, \mu_{j}>0, j=1$, $2, \cdots, N$. There may be other poles in the right half plane, but, wherever they are, their imaginary parts do not tend to zero as the damping tends to zero. Call these $\lambda_{i}^{\prime}=\nu_{i}^{\prime}+i \mu_{i}^{\prime}$. We concentrate on $\lambda_{i}$. Using [6, p. 212]

$$
H_{0}^{(2)}(\xi)=\left(\frac{2}{\pi \xi}\right)^{1 / 2} \exp [-i(\xi-\pi / 4)]+O\left(\xi^{-3 / 2}\right),
$$

we find, for the residue at $\lambda_{i}$,

$$
\begin{aligned}
R_{i}=\left[\frac{2}{\pi r}\left(\nu_{j}-i \mu_{j}\right)\right]^{1 / 2} \exp \left[-i\left(r \nu_{i}-\pi / 4\right)\right] & \\
\cdot \exp \left(-r \mu_{j}\right) & \frac{N\left(\lambda_{j} ; k_{1}, k_{2}\right)}{\left.\frac{\partial}{\partial \lambda} D\left(\lambda ; k_{1}, k_{2}\right)\right|_{\lambda_{i}}}+O\left(r^{-3 / 2}\right) .
\end{aligned}
$$

The first term is exponentially attenuated but the constant $\mu_{i}$ is small for small damping. The term contains a factor $r^{-1 / 2}$, as contrasted with the $r^{-1}$ factor produced by the contour integrations. Thus this residue term can make the dominant contribution for moderate The relative contributions could be better estimated by a more careful study of the contour integrations, but we have not deemed this worthwhile, since we are primarily interested in the limiting case of zero damping. There $\mu_{i} \rightarrow 0$.

For any other poles $\lambda_{j}^{\prime}$ the exponential decay factor is exp $\left(-\mu_{j}^{\prime} r\right)$ and this remains effective when the damping goes to zero since the $\mu_{j}^{\prime}$ do not vanish.

Inasmuch as only a finite number of poles of the integrand lie within the contour used, and this number, by virtue of Hurwitz's theorem, must remain finite as $\gamma_{1}$ and $\gamma_{2} \rightarrow 0$, we may pass to the limit in our final results, and get

$$
\begin{aligned}
\phi_{1}(r, 0, t)=-2 i\left(\frac{2 \pi}{r}\right)^{1 / 2} \sum_{i=1}^{N} \exp \left[-i\left(r \nu_{i}\right.\right. & -\omega t)] \\
& \cdot \frac{\nu_{j}^{1 / 2} N\left(\nu_{i} ; k_{1}(0), k_{2}(0)\right)}{\left.\frac{\partial}{\partial \lambda} D\left(\lambda ; k_{1}(0), k_{2}(0)\right)\right|_{\nu_{i}}}+\frac{A(R, r)}{r},
\end{aligned}
$$

where $A(R, r)$ is bounded in $r$.

In case $k_{1}(0)<k_{2}(0)$ the sum is empty. 
7. Final remarks and mathematical retrospect. The form of (6.19) is the customary one for such problems. It should be noted that $\phi_{1}$ does not, in the undamped case, satisfy the conditions sufficient for the applicability of the Hankel transform. Thus, previous ambiguities experienced in dealing with this kind of problem may be traced directly to the improper use of the transform method. The introduction of the damping term, whether one views it as a mathematical fiction or a physical reality, is a convenient way of avoiding these difficulties.

Finally, it should be remarked that Eq. (6.19) strongly suggests that no poles can occur in the upper right portion of the $\lambda$-plane, since such a pole would give rise to a term $\exp \left[i\left(r \nu_{i}^{\prime}+\omega t\right)\right] \exp \left(-\mu_{i}^{\prime} r\right)$ representing a converging wave. There is no physical mechanism for producing such waves.

Acknowledgments. The author is indebted to I. I. Kolodner, W. J. Byatt, and M. J. Norris for many helpful suggestions and discussions concerning this problem.

\section{BIBLIOGRAPHY}

1. C. L. Pekeris, Theory of propagation of sound in a half space of variable sound velocity, J. Acoust. Soc. Amer. 18, 295 (1946)

2. C. L. Pekeris, Theory of propagation of explosive sound in shallow water, Geol. Soc. Amer. Memoir 27, 1948

3. I. Rudnick, The propagation of an acoustic wave along a boundary, J. Acoust. Soc. Amer. 18, 348 (1947)

4. N. A. Haskell, Asymptotic approximation for the normal modes in sound channel wave propagation, J. Appl. Phys. 22, 157 (1951)

5. I. N. Sneddon, Fourier transforms, McGraw-Hill, New York, 1951

6. G. N. Watson, $A$ treatise on the theory of Bessel functions, Cambridge Press, 1958

7. E. C. Titchmarsh, The Theory of functions, Oxford Press, 1952

8. H. Lamb, On the propagation of tremors over the surface of an elastic solid, Phil. Trans. Roy. Soc. 203, 1 (1904) 